\title{
Patterned laser annealing of silicon oxide films
}

\author{
J. Richter $\cdot$ J. Meinertz $\cdot$ J. Ihlemann
}

Received: 13 August 2010 / Accepted: 8 April 2011 / Published online: 19 May 2011

(c) Springer-Verlag 2011

\begin{abstract}
UV-absorbing silicon monoxide $\left(\mathrm{SiO}_{x}, x \approx 1\right)$ thin films on fused silica substrates are irradiated by an ArF excimer laser (wavelength $193 \mathrm{~nm}$ ) in the sub-ablation threshold regime. Multi-pulse irradiation of films with $\sim 200$-nm thickness at a fluence of about $100 \mathrm{~mJ} / \mathrm{cm}^{2}$ leads to a significant increase of the UV transmission, indicating the oxidation of $\mathrm{SiO}_{x}$ to $\mathrm{SiO}_{2}$. The quality of the obtained films after this laser annealing process depends on the oxygen content of the environment. Irradiation in air at atmospheric pressure leads to the formation of sub-micron-sized oxide particles on top of the film. Structured illumination is applied either to form areas of the film with changed transmission and refractive index, or for the formation of regular particle patterns with sub-micron periods. These processes can be utilized for the fabrication of phase masks or for various types of surface functionalization.
\end{abstract}

\section{Introduction}

Silicon sub-oxide $\left(\mathrm{SiO}_{x}, x<2\right)$ or silicon monoxide $(\mathrm{SiO})$ layers are used for various applications. For example, they are employed as part of multi-layer coatings in the infrared range [1]. They serve as antireflective layers or as protective coatings against corrosion. In electronics, $\mathrm{SiO}$ is used e.g. as a dielectric material for thin-film capacitors [2]. During the last 10 years a new application field has evolved: $\mathrm{SiO}_{x}$ is used as the source material for the generation of $\mathrm{Si}$ nanocrystals to be used as integrated light emitters in the

J. Richter · J. Meinertz $\cdot$ J. Ihlemann $(\bowtie)$

Laser-Laboratorium Göttingen e.V., Hans-Adolf-Krebs-Weg 1,

37077 Göttingen, Germany

e-mail: juergen.ihlemann@llg-ev.de

Fax: +49-551-503599 field of silicon nanophotonics [3, 4]. For example, by depositing $\mathrm{SiO}_{x}$ layers just a few $\mathrm{nm}$ thin and heating them in inert atmosphere, material segregation leads to the formation of Si nanocrystals of a well-defined size [5].

But there is another field of application using $\mathrm{SiO}_{x}$ as a precursor material to fabricate $\mathrm{SiO}_{2}$ components. Heating of $\mathrm{SiO}_{x}$ in air at sufficiently high temperatures leads to conversion into $\mathrm{SiO}_{2}$. This can be utilized for the laser-based micro-patterning of $\mathrm{SiO}_{2}$. $\mathrm{SiO}_{2}$ itself is difficult to pattern by laser ablation with high resolution due to its lack of optical absorption from the deep UV to the near IR, so that direct coupling of laser light into the material is impeded. In contrast, $\mathrm{SiO}_{x}$ is strongly UV absorbing and can therefore be processed very well by UV-laser ablation. After creating a desired ablation pattern in $\mathrm{a} \mathrm{SiO}_{x}$ layer deposited on a fused silica substrate, the remaining $\mathrm{SiO}_{x}$ is oxidized to $\mathrm{SiO}_{2}$ leading to a monolithic patterned silica component. In this way, pure silica phase masks and diffractive optical elements have been successfully fabricated [6].

The oxidation of $\mathrm{SiO}_{x}$ to $\mathrm{SiO}_{2}$ by heating the samples in a furnace (thermal annealing) can be controlled well, but this method exhibits some drawbacks. As a rather high temperature $(>1000 \mathrm{~K})$ is required, only substrates resisting such high temperatures (silicon and fused silica) can be used. Furthermore, the oxidation process cannot be applied in a spatially resolved way by this method. Laser annealing, i.e. laser-induced oxidation of $\mathrm{SiO}_{x}$ to $\mathrm{SiO}_{2}$, could be a method solving both problems. Due to its capability of localized action, the substrate is not heated substantially, and a patterned oxidation should be possible giving rise to a lot of new applications.

In former attempts of laser annealing, spatial resolution has not been addressed so far. Blum et al. demonstrated photo-oxidation of $\mathrm{SiO}$ films with multi-pulse 193-nm-laser radiation [7]. Gallas et al. used single excimer laser pulses 
at $248 \mathrm{~nm}$ for annealing and pointed out that there is only a narrow window of fluence values where $\mathrm{SiO}_{x}$ is annealed without being ablated [8]. Fogarassy et al. showed that in the case of UV-laser irradiation of $\mathrm{SiO}_{x}$ layers the thermal contribution seems of primary importance in explaining the photo-oxidation [9]. It has been confirmed that oxidation in fact produces $\mathrm{SiO}_{2}$ [10]. However, in all these papers, the quality of the annealed films with respect to optical applications is not discussed.

In this paper we investigate the conditions for obtaining good quality oxide films by laser annealing and demonstrate the possibility of a patterned (spatially selective) oxidation process. In contrast to other methods for spatially selective surface functionalization like chemical or plasma treatments, which require a number of process steps like deposition of a mask layer, patterning of the mask, an etch or plasma process, mask removal, etc., this treatment is accomplished in a single process step.

\section{Experiment}

$\mathrm{SiO}_{x}$ films of $200-300 \mathrm{~nm}$ thickness with $x \approx 1$ were deposited on 2-mm-thick fused silica substrates by vacuum evaporation (Laseroptik Garbsen, Germany). These coatings are hard, exhibit good adhesion, and are chemically stable at room temperature. In contrast to $\mathrm{SiO}_{2}$, they absorb strongly in the ultraviolet spectral range. The absorption coefficient of $\mathrm{SiO}_{x}$ at $193 \mathrm{~nm}$ is about $4.6 \times 10^{5} \mathrm{~cm}^{-1}$ for $x \approx 1$; the refractive index is about $n=1.9$ [11].

Irradiation was performed with an ArF excimer laser (Lambda Physik LPX 315) emitting at 193 nm. Laser fluence was adjusted using a variable attenuator. Homogeneous irradiation of a field of $5.5 \mathrm{~mm} \times 5.5 \mathrm{~mm}$ was obtained by using a beam homogenizer consisting of crossed cylindrical lens arrays (focal length: $100 \mathrm{~mm}$, pitch: $4 \mathrm{~mm}$ ) in combination with a spherical Fourier lens of $250 \mathrm{~mm}$ focal length.

For structured irradiation, the laser illuminates a $\mathrm{Cr}$-onquartz mask containing a linear or crossed grating pattern. The mask is imaged onto the sample using either a fused silica lens for medium resolution or a Schwarzschild objective $(25 \times$ demagnification, 0.4 numerical aperture) to obtain high resolution.

For experiments in different gas environments, the sample is mounted in a housing that can be flushed with nitrogen (purity 99.999\%) or argon (purity 99.998\%) at atmospheric pressure.

Absorption spectra were recorded with a Perkin Elmer Lambda 19 spectrophotometer. The surface morphology was inspected by a Zeiss DSM 962 scanning electron microscope (SEM). Spatially resolved transmission of the annealed samples at $193 \mathrm{~nm}$ was measured by illuminating them with the ArF laser at low intensity and imaging the sample surface (magnified) onto the UV-sensitive screen of a beam profiler camera.

\section{Results}

Figure 1a displays transmission spectra of a 262-nm-thick $\mathrm{SiO}_{x}$ film irradiated in air. Before irradiation, the onset of absorption below $400 \mathrm{~nm}$ can be clearly seen. After irradiation with 10,000 pulses at $120 \mathrm{~mJ} / \mathrm{cm}^{2}$ the apparent UV transmission has slightly increased and the transmission in the visible has decreased. But this spectrum is strongly disturbed by a granular layer that has formed on the surface of the sample (Fig. 2a), leading to broadband scattering losses in the spectrometer. After cleaning the surface of this covering using a $\mathrm{KOH}$-based agent, the transmission is higher than $70 \%$ over the whole UV and visible spectrum. However, the thickness of the remaining film after removal of the granular covering is only about $100 \mathrm{~nm}$ as measured by a Dektak profilometer.

A different behavior is observed when irradiating the $\mathrm{SiO}_{x}$ films in nitrogen. Figure $1 \mathrm{~b}$ displays transmission spectra of a 223-nm-thick $\mathrm{SiO}_{x}$ film irradiated under nitrogen flushing. The UV transmission increases steadily with growing pulse number. In this case there is no covering layer present even after 50,000 pulses (Fig. 2b). The thickness in the irradiated area has decreased from $223 \mathrm{~nm}$ to about 180 nm. Performing the same procedure using Ar instead of $\mathrm{N}_{2}$ leads to nearly identical results (Fig. 1c), confirming the assumption that nitrogen behaves as inert gas and does not lead to a reaction with the $\mathrm{SiO}_{x}$.

The results of structured irradiation with medium resolution are shown in Fig. 3. The transmission at $193 \mathrm{~nm}$ is displayed in a false color representation. Extended areas of $5 \mathrm{~mm} \times 5 \mathrm{~mm}$ size with homogeneous transmission at 193 $\mathrm{nm}$ are obtained by irradiation in nitrogen with a homogenized beam. Figure 3a displays an array of four such areas each irradiated with 10,000 pulses. A pattern of $25-\mu \mathrm{m}$-wide stripes of alternatively high and low transmission is shown in Fig. 3b. In this case, the sample has been irradiated using a Cr-on-quartz mask with 50 - $\mu$ m-wide transparent stripes, which was imaged with 2:1 demagnification onto the $\mathrm{SiO}_{x}$ film. The irregularities in this pattern are due to the inhomogeneous beam and the above-mentioned grain formation as a consequence of creating the pattern in air.

A SEM image of an irradiation pattern with sub-micron periodicity generated in air is shown in Fig. 4. In this case, the four first-order diffracted beams of a crossed grating mask were recombined in the image plane of the Schwarzschild objective on the sample surface, leading to a two-dimensional interference pattern. The grains develop exactly at the positions of peak intensity. 
Fig. 1 Transmission spectra of laser-annealed $\mathrm{SiO}_{x}$ films:

(a) ArF-laser irradiation in air at $120 \mathrm{~mJ} / \mathrm{cm}^{2}, 10 \mathrm{~Hz}, \mathrm{SiO}_{x}$-layer thickness $262 \mathrm{~nm}$, (b) ArF-laser irradiation in nitrogen at $120 \mathrm{~mJ} / \mathrm{cm}^{2}, 10 \mathrm{~Hz}, \mathrm{SiO}_{x}$-layer thickness $223 \mathrm{~nm}$, (c) ArF-laser irradiation in argon at

$120 \mathrm{~mJ} / \mathrm{cm}^{2}, 10 \mathrm{~Hz}, \mathrm{SiO}_{x}$-layer thickness $223 \mathrm{~nm}$

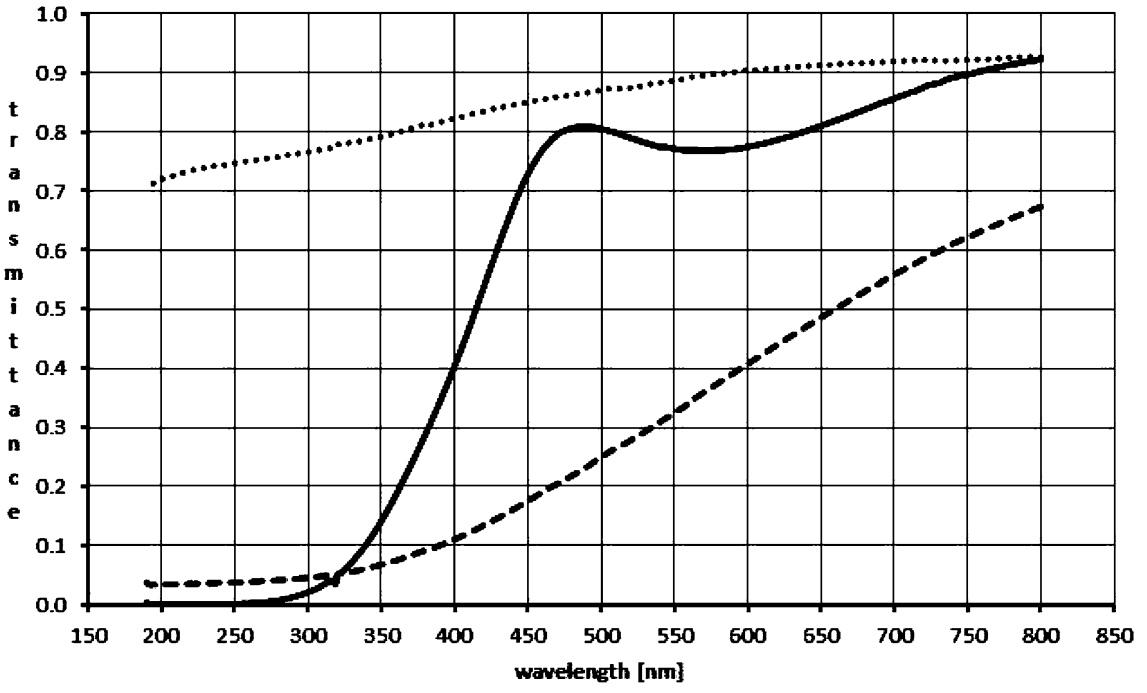

a prior to radiation $\quad$ - - after 10000 pulses $\quad \ldots .$. after cleaning

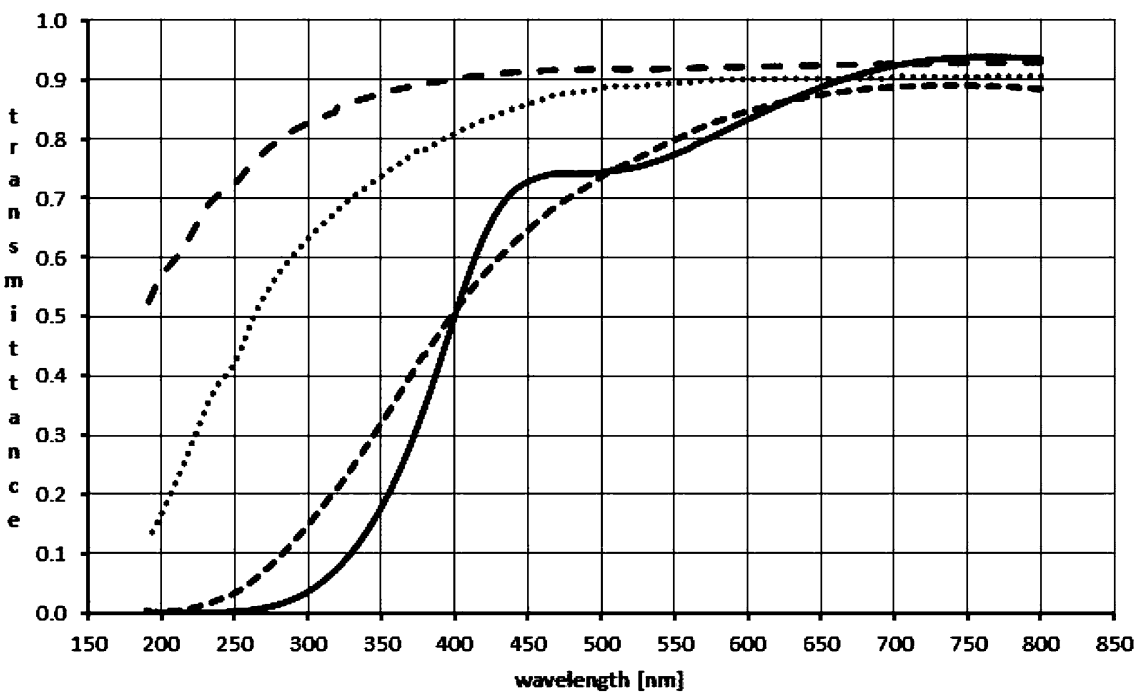

b — prior to irradiation

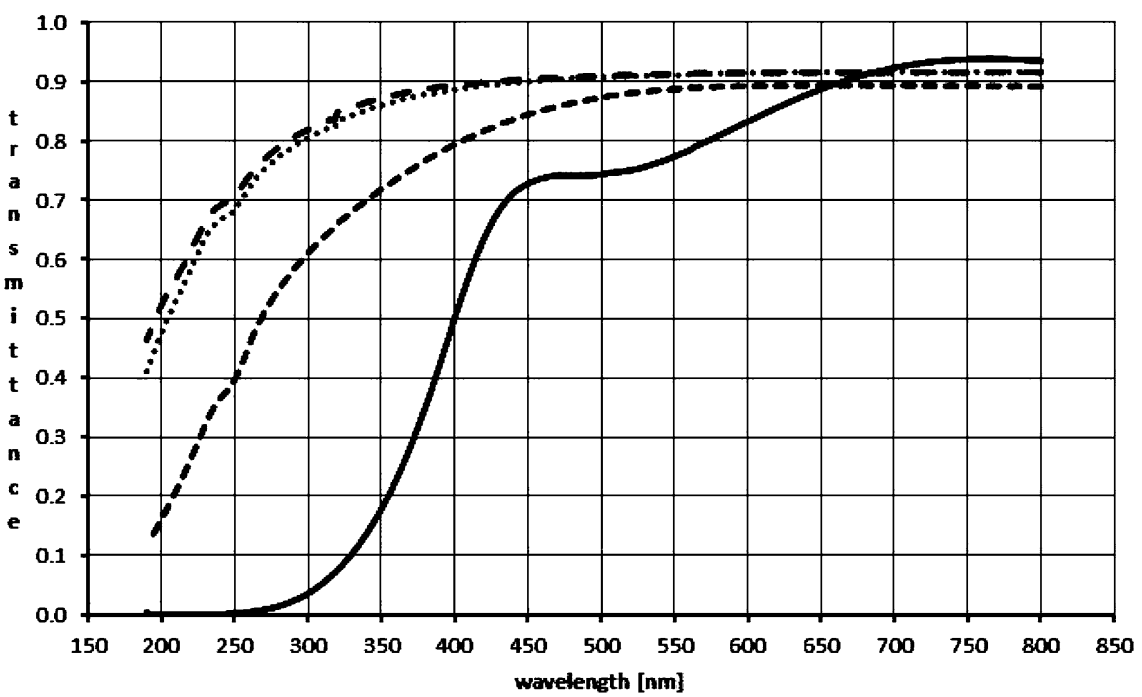

c - priorto irradiation - - after 1000 pulses ...... after 10000 pulses - - after 20000 pulses 

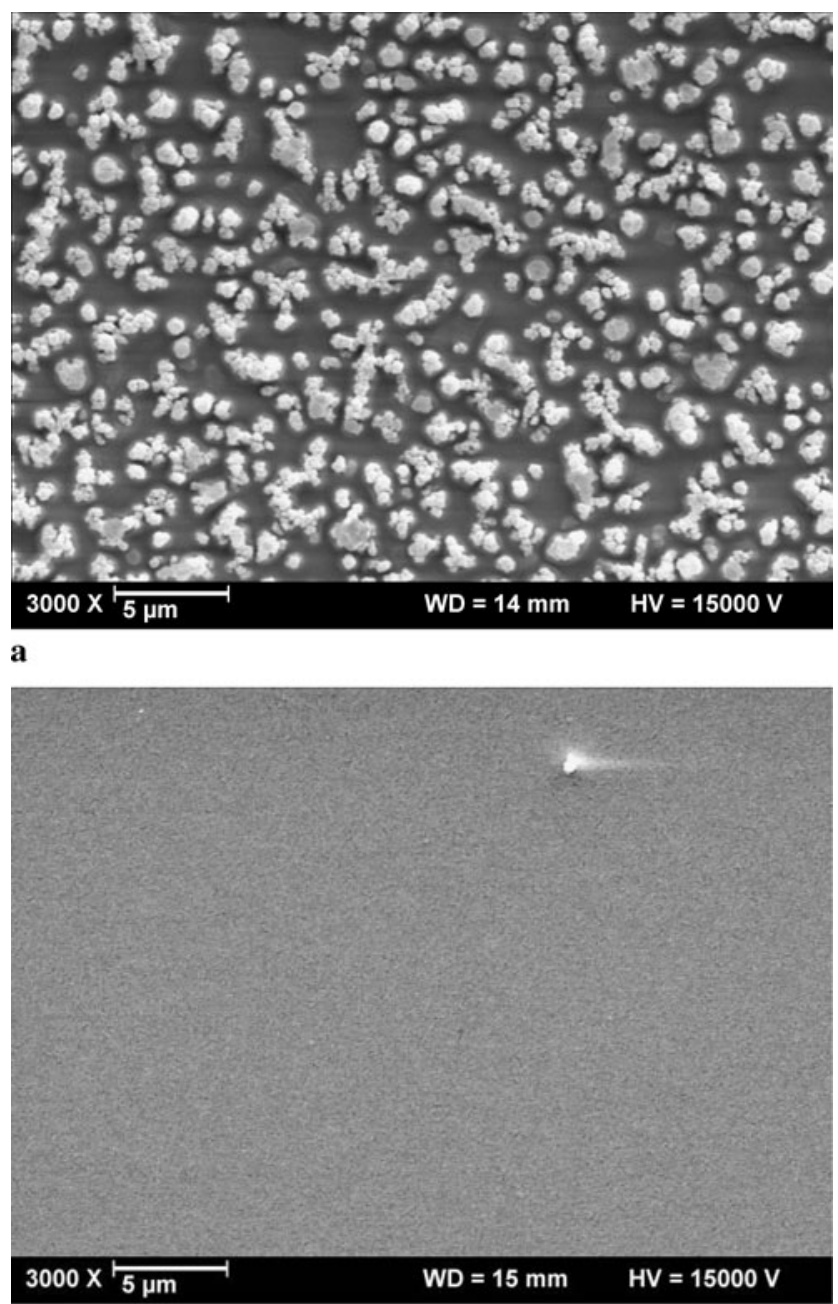

b

Fig. 2 SEM images of a 406-nm-thick $\mathrm{SiO}_{x}$ layer irradiated at 193 $\mathrm{nm}$ at $120 \mathrm{~mJ} / \mathrm{cm}^{2}$ : (a) irradiation in air, 80,000 pulses, (b) irradiation in nitrogen, 50,000 pulses

\section{Discussion}

The result of laser-induced oxidation of $\mathrm{SiO}_{x}$ is quite different from that obtained by furnace annealing. Furnace annealing for $8 \mathrm{~h}$ at $1200 \mathrm{~K}$ in air leads to highly transparent films with optical quality and a thickness of about 1.3-1.4 times the original $\mathrm{SiO}_{x}$-film thickness [12]. Laser annealing instead leads to a reduction of the film thickness, apart from the formation of the granular covering in air.

A pure photochemical effect has been observed in the case of laser-induced oxidation of silicon at $157 \mathrm{~nm}$ [13]. In this case, photochemical dissociation of molecular oxygen leads to oxygen atoms or ions, which rapidly diffuse through the already formed oxide layer. The thickness increase of the oxide layer is then limited by the recombination of oxygen atoms. This process cannot explain our results for two reasons: first, the wavelength of $193 \mathrm{~nm}$ is less effective concerning the dissociation of oxygen; second, the

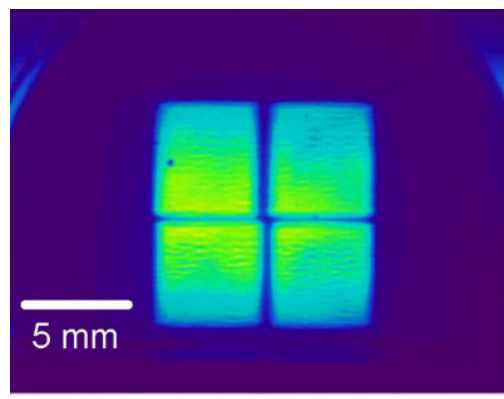

$\mathbf{a}$

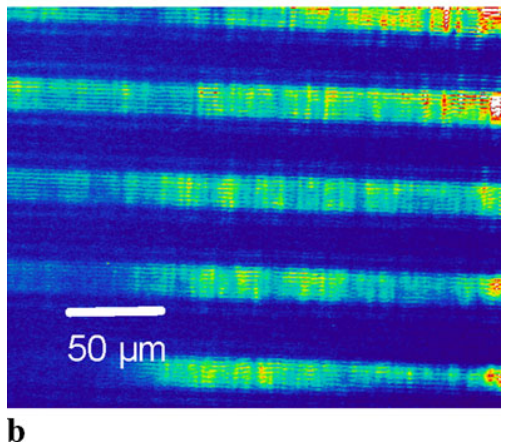

Fig. 3 UV transmission of patterns made by structured laser annealing and recorded with a UV-sensitive camera: (a) 223-nm $\mathrm{SiO}_{x}$, $193 \mathrm{~nm}, 120 \mathrm{~mJ} / \mathrm{cm}^{2}, 10,000$ pulses in nitrogen (beam-profile camera positioned directly behind the sample), (b) 144-nm $\mathrm{SiO}_{x}, 193 \mathrm{~nm}$, $80 \mathrm{~mJ} / \mathrm{cm}^{2}, 10,000$ pulses in air (beam-profile camera positioned in the image plane of a $10 \times$ magnifying microscope objective to record the intensity pattern at sufficiently high magnification)

thickness of $200 \mathrm{~nm}$ is far above the limit of oxygen atom diffusion without recombination [13]. This is confirmed by the results of irradiation with 180,000 pulses at low fluence $\left(20 \mathrm{~mJ} / \mathrm{cm}^{2}\right)$, leading to no noticeable change [14]. Furthermore, photochemical oxidation of thick $\mathrm{SiO}_{x}$ films using a mercury lamp was only successful in the case of films deposited slowly at comparatively high oxygen pressures, where oxygen is gettered during deposition and embedded in the film [15]. Dense films deposited rapidly at low oxygen pressure could not be oxidized in this way.

Thus, the heating of the $\mathrm{SiO}_{x}$ film seems to be essential for the oxidation process. On the one hand, in the laser process, the total duration of the film being at high temperature $(>1000 \mathrm{~K})$ is just a few milliseconds even when applying 50,000 pulses, so that slow processes effective in furnace heating can be excluded, as already Gallas et al. have pointed out [8]. On the other hand, the peak temperature reached during each pulse is much higher than $1200 \mathrm{~K}$ used in the case of furnace annealing.

According to a simple thermal model, the melting point of $\mathrm{SiO}_{x}$ of $1700 \mathrm{~K}$ is reached throughout the film when a 193-nm-laser pulse of $100 \mathrm{~mJ} / \mathrm{cm}^{2}$ is applied on a 150nm-thick $\mathrm{SiO}_{x}$ film [16]. This may explain the comparably fast oxidation within $10^{3}$ to $10^{5}$ laser pulses, if the concept of Liu et al. [17] for laser-induced oxidation of amor- 

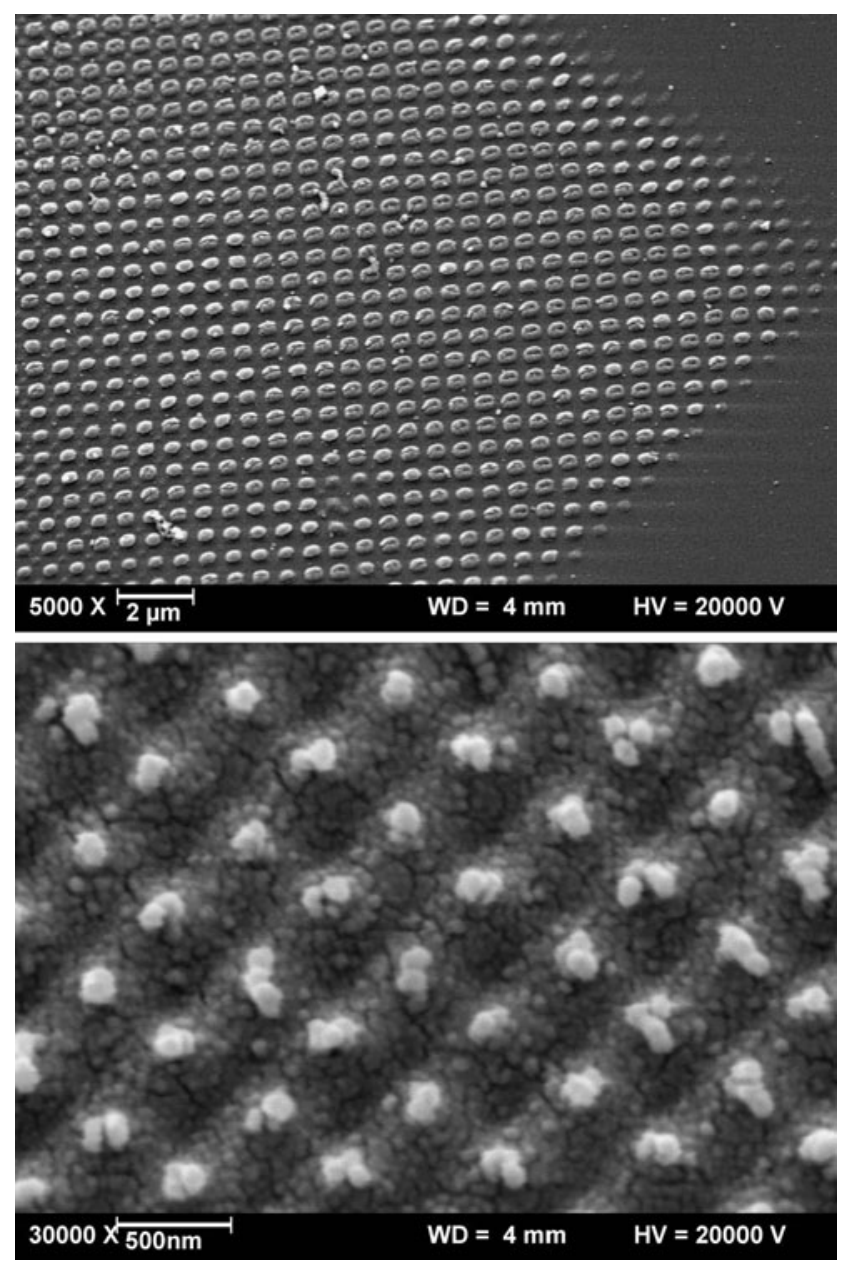

Fig. 4 SEM images of a particle pattern generated by structured irradiation of a 400-nm-thick $\mathrm{SiO}_{x}$ film in air; laser parameters: $193 \mathrm{~nm}$, $100 \mathrm{~mJ} / \mathrm{cm}^{2}, 5 \mathrm{~Hz}, 2000$ pulses

phous silicon is adopted here for $\mathrm{SiO}_{x}$ : in oxygen or air atmosphere of $1 \mathrm{~atm}$, oxygen molecules are very rapidly adsorbed on the surface. The laser pulse then melts the film followed by rapid diffusion of oxygen in the molten layer. At a high solid-liquid interfacial regrowth velocity, the adsorbed molecules are trapped in the regrown zone leading to deep oxidation [17].

The formation of grains may then be due to an excess supply of oxygen, leading to inhomogeneities that cannot be compensated for by successive laser pulses. Homogeneous irradiation then leads to arbitrarily positioned grain formation; patterned illumination causes preferential grain formation at high-intensity positions. A similar behavior has been observed for $\mathrm{KrF}$ laser annealing of silicon nanocrystal films prepared by pulsed laser deposition [10]: after a few pulses of $50 \mathrm{~mJ} / \mathrm{cm}^{2}$ applied in air, nanoparticles with sizes of 10$50 \mathrm{~nm}$ are formed. These particles are recrystallized from the film at positions of high intensity and are oxidized by successive annealing pulses. Also, for amorphous hydrogenated silicon sub-oxide films, laser irradiation at $355 \mathrm{~nm}$ with 120
$\mathrm{mJ} / \mathrm{cm}^{2}$ leads to 'pile-up'-formation of particles just at the high-intensity positions [18].

Perhaps this rapid oxidation is the reason of volume increase and formation of the granular crust. If the process is slowed down due to limited supply of oxygen (nitrogen or argon atmosphere with traces of oxygen), repetitive heating leads to relaxation preserving the original film structure. However, apparently even below the threshold of ablation some desorption takes place (of the order of $10^{-3}$ $\mathrm{nm} /$ pulse), leading to the observed diminished film thickness after multi-pulse irradiation. In any case, a significant change of the refractive index from $n=1.9$ of $\mathrm{SiO}_{x}$ to about $n=1.5$ of $\mathrm{SiO}_{2}$ is connected with this oxidation process.

\section{Conclusion}

Spatially selective oxidation of $\mathrm{SiO}_{x}$ layers is possible by excimer laser irradiation. In a strongly diluted oxygen atmosphere smooth films with optical quality are obtained. This type of processing is suitable e.g. for the fabrication of optical phase elements, which combine a smooth surface and variations in the refractive index. In an oxygen-rich environment rapid oxidation of $\mathrm{SiO}_{x}$ films occurs. In combination with high-resolution irradiation, this can be utilized for the fabrication of $\mathrm{SiO}_{2}$-nanoparticle arrays. In combination with a metalization, these nanoparticle arrays are promising candidates for surface-enhanced effects. Applications may include e.g. surface-enhanced Raman spectroscopy (SERS).

Acknowledgements Financial support by the German Ministry of Economics and Technology is gratefully acknowledged (Grant No. 16IN0505).

\section{References}

1. N. Kaiser, H.K. Pulker (eds.), Optical Interference Coatings (Springer, Berlin, 2003)

2. P.J. Harrop, D.S. Campbell, Thin Solid Films 2, 213 (1968)

3. L. Khriachtchev (ed.), Silicon Nanophotonics (World Scientific, Singapore, 2009)

4. L. Pavesi, Adv. Opt. Technol. 2008, 416926 (2008)

5. L. Yi, R. Scholz, M. Zacharias, J. Lumin. 122-123, 750 (2007)

6. M. Schulz-Ruhtenberg, J. Ihlemann, J. Heber, Appl. Surf. Sci. 248, 190 (2005)

7. S.E. Blum, K.H. Brown, R. Srinivasan, Appl. Phys. Lett. 43, 1026 (1983)

8. B. Gallas, C.-C. Kao, S. Fisson, G. Vuye, J. Rivory, Y. Bernard, C. Belouet, Appl. Surf. Sci. 185, 317 (2002)

9. E. Fogarassy, S. Unamuno, J.L. Regolini, C. Fuchs, Philos. Mag., B 55, 253 (1987)

10. X.Y. Chen, Y.F. Lu, Y.H. Wu, B.J. Cho, B.J. Yang, T.Y.F. Liew, J. Vac. Sci. Technol. B 22, 1731 (2004)

11. M. Jahn, J. Richter, R. Weichenhain-Schriever, J. Meinertz, J. Ihlemann, Appl. Phys. A 101, 533 (2010)

12. J. Ihlemann, R. Weichenhain-Schriever, J. Laser Micro Nanoeng. 4, 100 (2009) 
13. A.V. Osipov, P. Patzner, P. Hess, Appl. Phys. A 82, 275 (2006)

14. M. Schulz-Ruthenberg, Diploma thesis, University of Göttingen, 2003

15. A.P. Bradford, G. Hass, M. McFarland, E. Ritter, Appl. Opt. 4, 971 (1965)
16. M. Jahn, Bachelor thesis, Technical University of Ilmenau, 2009

17. Y.S. Liu, S.W. Chiang, F. Bacon, Appl. Phys. Lett. 38, 1005 (1981)

18. A. Janotta, Y. Dikce, M. Schmidt, C. Eisele, M. Stutzmann, J. Appl. Phys. 95, 4060 (2004) 